\title{
Cigarette Smoking Progression Among Young Adults Diagnosed With ADHD in Childhood: A 16-year Longitudinal Study of Children With and Without ADHD
}

\author{
John T. Mitchell PhD, ${ }^{1}$ Andrea L. Howard PhD, ${ }^{2}$ \\ Katherine A. Belendiuk PhD, ${ }^{3}$ Traci M. Kennedy PhD, ${ }^{4}$ \\ Annamarie Stehli MPH, ${ }^{5}$ James M. Swanson PhD, ${ }^{5}$ Lily Hechtman MD, ${ }^{6}$ \\ L. Eugene Arnold MD, ${ }^{7}$ Betsy Hoza PhD, ${ }^{8}$ Benedetto Vitiello MD, ${ }^{9}$ \\ Bo Lu PhD, ${ }^{10}$ Scott H. Kollins PhD, ${ }^{1}$ Brooke S.G. Molina PhD ${ }^{11}$
}

'Department of Psychiatry \& Behavioral Sciences, Duke University Medical Center, Durham, NC; ${ }^{2}$ Department of Psychology, Carleton University, Ottawa, Ontario, Canada; ${ }^{3}$ Genentech, South San Francisco, CA; ${ }^{4}$ Department of Psychiatry, University of Pittsburgh School of Medicine, Pittsburgh, PA; ${ }^{5}$ Department of Pediatrics, University of California, Irvine, CA; ${ }^{6}$ Division of Child Psychiatry, McGill University, Montreal Children's Hospital, Montreal, Quebec, Canada; ${ }^{7}$ Department of Psychiatry and Behavioral Health, Ohio State University, Columbus, $\mathrm{OH}$; ${ }^{8}$ Department of Psychological Science, University of Vermont, Burlington, VT; ${ }^{9}$ Division of Child and Adolescent Neuropsychiatry, University of Turin, Italy; ${ }^{10}$ Division of Biostatistics, The Ohio State University, Columbus, OH; ${ }^{11 D e p a r t m e n t s ~ o f ~}$ Psychiatry, Psychology, and Pediatrics, University of Pittsburgh, Pittsburgh, PA

Corresponding Author: John T. Mitchell, PhD, Department of Psychiatry \& Behavioral Sciences; Duke University Medical Center; 2608 Erwin Road; Pavilion East; Suite 300; Durham, NC, 27705, USA. Telephone: 919-681-0012; Fax: 919-681-0016;

E-mail: john.mitchell@duke.edu

\begin{abstract}
Introduction: Children with attention-deficit/hyperactivity disorder (ADHD) are at increased risk for smoking cigarettes, but there is little longitudinal research on the array of smoking characteristics known to be prognostic of long-term smoking outcomes into adulthood. These variables were studied into early adulthood in a multisite sample diagnosed with ADHD combined type at ages 7-9.9 and followed prospectively alongside an age- and sex-matched local normative comparison group (LNCG).

Methods: Cigarette smoking quantity, quit attempts, dependence, and other characteristics were assessed in the longitudinal MultimodalTreatment Study of Children with ADHD (MTA) eight times to a mean age of 24.9 years: ADHD $n=469$; LNCG $n=240$.

Results: In adulthood, the ADHD group had higher rates of daily cigarette smoking, one or more quit attempts, shorter time to first cigarette of the day, and more severe withdrawal than the LNCG. The ADHD group did not appear to have better smoking cessation rates despite a higher proportion quitting at least once. Smoking quantity and nicotine dependence did not differ between groups. The ADHD group reported younger daily smoking onset and faster progression from smoking initiation to daily smoking across assessments. Finally, ADHD symptom severity in later adolescence and adulthood was associated with higher risk for daily smoking across assessments in the ADHD sample.
\end{abstract}


Conclusions: This study shows that ADHD-related smoking risk begins at a young age, progresses rapidly, and becomes resistant to cessation attempts by adulthood. Prevention efforts should acknowledge the speed of uptake; treatments should target the higher relapse risk in this vulnerable population.

Implications: Although childhood ADHD predicts later smoking, longitudinal studies of this population have yet to fully characterize smoking behaviors into adulthood that are known to be prognostic of long-term smoking outcome. The current study demonstrates earlier and faster progression to daily smoking among those with a childhood ADHD diagnosis, as well as greater risk for failed quit attempts. Prevention efforts should address speed of smoking uptake, while treatments are needed that address smoking relapse risk. The current study also demonstrates ADHD symptom severity over development increases daily smoking risk, implicating the need for continuous ADHD symptom management.

\section{Introduction}

Attention-deficit/hyperactivity disorder (ADHD) is a childhoodonset disorder characterized by pervasive and functionally impairing inattention and/or hyperactivity-impulsivity. ${ }^{1}$ Children with ADHD are more likely to smoke cigarettes in adolescence ${ }^{2-4}$ and adulthood, ${ }^{5-11}$ but little prospective longitudinal research has fully characterized their prognostically important smoking behaviors into adulthood when smoking patterns become more firmly established. ${ }^{12}$ As a result, little is known about the specific characteristics of smoking (eg, quantity, success in quitting) as smokers with childhood ADHD become adults. One prospective study recently reported group differences in smoking characteristics as a function of childhood ADHD (eg, younger age of smoking initiation, faster progression to regular smoking), but nearly half of the sample was under 18 years old with a mean age of 17.75 years. ${ }^{13}$ As such, failure to find other group differences (eg, quit attempts) may have been hampered by a limited number of adults with prolonged smoking histories. Indeed, the mean age of daily smoking onset is 18.2 years with about one-third of smokers progressing to daily smoking after age $18 .{ }^{14}$ Studies that examine a broad array of smoking outcomes in adults are needed to better capture childhood ADHD smoking outcomes that do not fully emerge until adulthood. The current study addresses this need with a prospectively studied multisite sample of children with ADHD followed to their mid-20s.

Although ADHD persists into adulthood for about $60 \%$ of children with $\mathrm{ADHD}^{15}$ and places individuals at risk for worse substance use outcomes, ${ }^{16}$ the impact of ADHD symptom persistence over time with cigarette smoking is an understudied area. Persistent ADHD was not associated with daily smoking in the Rhodes and colleagues sample, ${ }^{13}$ but this question needs to be examined in an adult sample. Moreover, a dimensional approach to symptoms may provide a more sensitive analysis for daily smoking given the potential functional importance of subthreshold symptom persistence for ADHD. ${ }^{17,18}$ The current study provides an important contribution by being the first to examine ADHD symptom severity over adolescence and into adulthood in relation to daily smoking — a smoking variable associated with significantly higher risk of adult death. ${ }^{19}$

The longitudinal follow-up of the children in the Multimodal Treatment study of ADHD (MTA) ${ }^{20}$ repeatedly assessed cigarette smoking through adolescence into early adulthood when participants were about 25 years old. It therefore provides an opportunity to address the aforementioned gaps in the literature. The MTA began as a 14-month randomized controlled trial of treatments for children with ADHD and continued as a prospective observational study with regular assessments for 16 years. The MTA features a well-characterized, large multisite sample and a local normative comparison group (LNCG) of classmates matched for age and sex. ${ }^{20}$

Previous publications from the MTA demonstrate the high rates of daily smoking in late adolescence ${ }^{21,22}$ and adulthood ${ }^{23}$ in the ADHD group compared to the LNCG. The MTA therefore provides an excellent opportunity to determine whether prognostically relevant smoking characteristics, such as high quantity of cigarette consumption, greater nicotine dependence, and failed quit attempts, are related to childhood ADHD. Our first aim was to compare a variety of young adult smoking outcomes (ie, smoking quantity, nicotine dependence, quit attempts, a smoking relapse indicator variable, and withdrawal symptoms) between those with and without childhood ADHD. Our second aim was to compare time to the following events between those with and without childhood ADHD: smoking initiation, daily smoking onset, and progression from initiation to daily smoking. Across both of these aims, we predicted worse smoking outcomes among those with childhood ADHD compared to the LNCG, including earlier onset and more rapid smoking progression for the ADHD group than the LNCG. Our third aim was to examine the association between ADHD symptom severity at different developmental stages (eg, adolescence and adulthood) and the probability of daily smoking over time within the ADHD sample. We predicted a positive association between ADHD symptom severity and daily smoking through adolescence and early adulthood within the ADHD group.

\section{Methods}

\section{Participants}

Participants were from the longitudinal follow-up of the MTA. Children received a diagnosis of ADHD, Combined Type $(N=579)$ at study entry when they were 7.0-9.9 years old. ${ }^{20}$ At each of six sites, children were randomly assigned to one of four treatment groups: Medication Management alone, Behavioral Treatment alone, Combined Treatment, or Community-treated Comparison. ${ }^{24}$ Participants were assessed at completion of the 14-month treatment phase and again at 2, 3, 6, 8, 10, 12, 14, and 16 years after randomization. The LNCG ( $N=258$ without ADHD at study entry) was recruited at the 2-year assessment. ${ }^{23}$ Thus, data for the LNCG are available beginning when their mean age was 10.4 years $(S D=1.08)$. Details about group differences at 2 years are summarized elsewhere. ${ }^{25}$ From the original sample, $95 \%$ of ADHD group participants were reassessed at least once between the 2- and 16-year waves and were 
retained in our analyses. Retention in adulthood (assessed at least once in the three adult assessments at 12-, 14-, or 16-year follow-up) was 476 of 579 (82\%) for the ADHD group and 241 of 258 (93\%) for the LNCG. Participants with and without complete data in the MTA were not significantly different on most demographic variables and equally met missing-at-random criteria. ${ }^{26}$ See Swanson et al. ${ }^{27}$ for group differences in ADHD symptoms between the ADHD group and LNCG. The final sample included 469 ADHD and 240 LNCG participants who completed assessments of smoking behavior in at least one adult assessment. The most recent adult assessment was used.

\section{Measures}

\section{Tobacco Smoking}

Tobacco smoking was assessed at each assessment with the selfreported Substance Use Questionnaire ${ }^{22,28}$ (SUQ) adapted for the MTA. The SUQ assessed daily smoking in the past year, smoking amount in the past year (ie, about a half pack or more per day; about a full pack or more per day), quit attempts, and withdrawal symptoms (ie, craving, difficulty concentrating, and restlessness). For analyses involving the 12- through 16-year assessments, participants were coded as daily smokers if they smoked "once a day" or more in response to "In the past year, how often did you smoke cigarettes?" or if they responded "yes" to "Are you currently a daily smoker?" For the 2-through 10-year assessments, participants were coded as daily smokers if they smoked at least one cigarette per day in response to "During the past month, how many cigarettes have you smoked on an average day?" Responses to the restlessness question were followed by, “Are you normally a restless person (i.e., don't like to sit for long periods of time, need to move about frequently)?” on a 4-point Likert scale. We examined quit attempts among those who were ever weekly smokers for the 12- through 16-year assessments (i.e., those who responded "once a week or more" in response to "In the past year, how often did you smoke cigarettes?") given that there are significant health effects among such non-daily smokers. ${ }^{29}$ Smoking initiation age was the earliest age when smoking was first endorsed or retrospectively reported. Age of daily smoking onset was based on age at the time daily smoking was first reported. Latency to daily smoking was the difference between these two variables. Test-retest reliability of the SUQ smoking items is good (eg, kappa for ever tried cigarettes $=.87) \cdot{ }^{13}$

\section{Nicotine Dependence Severity}

The Fagerström Test for Nicotine Dependence (FTND) ${ }^{30}$ assessed nicotine dependence severity among daily smokers in adulthood. Total scores $\geq 5$ indicate moderate to high dependence. The FTND is both reliable and valid in cigarette-smoking samples. ${ }^{31,32}$ The first item from the FTND assesses time to first cigarette upon waking (ie, $\leq 5 \mathrm{~min}, 6-30 \mathrm{~min}, 31-60 \mathrm{~min}$, and $\geq 60 \mathrm{~min}$ ). This item was separately analyzed because it has predictive validity for smoking relapse during quit attempts and is an index of ability to quit smoking. ${ }^{33}$

\section{ADHD Symptom Severity}

ADHD symptom severity was assessed by collateral reporters (>80\% mothers) using common items from the Swanson, Nolan, and Pelham Rating Scale (SNAP) ${ }^{34}$ and Conners Adult ADHD Rating Scale (CAARS). ${ }^{35}$ These measures have adequate to excellent internal reliability ( $\alpha$ ranging .71-.93 for the SNAP ${ }^{36}$ and .74-.92 for the CAARS $^{35}$ ), as well as validity. ${ }^{35,37}$ The SNAP was administered 2 to 10 years after baseline for participants under age 18; the CAARS was administered at 8 to 16 years after baseline for participants age $\geq 18$. The 18 DSM-IV items from the SNAP were matched with parallel CAARS items. Response options ranged from 0 (not at all/ never) to 3 (very often/very frequently). Total symptom severity was calculated as the mean of all 18 item responses, whereas the inattentive and hyperactive-impulsive symptom severity scores were calculated as the means of their respective subscale items ( 9 items each).

\section{Data Analysis}

\section{Aims 1 and 2 Analytic Plan}

Adulthood smoking outcomes were compared between ADHD and LNCG using ANOVA and logistic regression. Progression of smoking initiation and daily smoking were examined via Cox proportional hazards regression. As we have done elsewhere, ${ }^{23,38}$ household disadvantage was accounted for by including weighted effects-coded variables comparing children from two-parent households and at least one college-educated parent to the sample average (bousehold advantage), and children from single-parent households with no college-educated parents to the sample average (household disadvantage). Presence of a baseline diagnosis of oppositional defiant disorder (ODD) or conduct disorder (CD) was covaried as we have done in previous studies, ${ }^{23}$ as well as sex $($ Female $=0$, Male $=1$ ) and race (dummy-coded: Black $=1$, All other $=0$; Hispanic/Other/Mixed ethnicity $=1$, All other $=0$ ).

\section{Aim 3 Analytic Plan}

The longitudinal association between ADHD symptom severity and probability of daily smoking in the ADHD group was tested via latent growth modeling in Mplus v.8.0. ${ }^{39}$ First, we established an appropriate model for change over time in daily smoking. Preliminary data inspection suggested that the probability of daily smoking increased through adolescence but leveled off in adulthood. We used a piecewise linear function of time ${ }^{40}$ with an inflection or "knot" point at the 12-year assessment to separately estimate rates of change in daily smoking through adolescence up to the 12-year follow-up and in early adulthood after the 12-year follow-up. Second, we estimated the association between ADHD symptom severity and daily smoking at each wave of assessment (a time-varying covariate relation), to determine whether more severe symptoms at specific points in time were linked to a greater chance of daily smoking at the same time. As in our analyses for Aims 1 and 2, household (dis)advantage, baseline ODD or CD, sex, and race were included as covariates. We also tested their effects on rates of change in daily smoking, but only retained statistically significant terms. Diagonally weighted least squares estimation was used to obtain parameter estimates and standard errors. Cases with missing values were retained and estimates were unbiased under a missing at random mechanism. ${ }^{41}$

\section{Results}

\section{Aim 1: Smoking Outcomes in Young Adulthood}

Table 1 summarizes ADHD and LNCG comparisons in young adulthood. Daily smoking over the past year, higher in the ADHD group than the LNCG, is reported in Molina et al. ${ }^{23}$ and repeated in Table 1 to facilitate interpretation of results $(p<.001)$. Quantity of cigarettes smoked in the past year was compared between ADHD and LNCG daily smokers. Statistically significant group differences were not found for either smoking about a half pack or more a day or for smoking a full pack or more daily. The rate of moderate-to-high nicotine dependence among daily 
Table 1. Smoking Outcome at Adult Follow-up

\begin{tabular}{|c|c|c|c|c|c|}
\hline & ADHD & LNCG & & & \\
\hline & $N(\%)$ & $N(\%)$ & $B$ & $p$ value & OR $(95 \% \mathrm{CI})$ \\
\hline Daily Smokers in the Past Year ${ }^{a}$ & $180(38.4 \%)$ & $46(19.2 \%)$ & 0.95 & $<.001$ & $2.59(1.76-3.81)$ \\
\hline Smoking about a Half Pack or More Daily in the Past Year & $126(70.0 \%)$ & $26(56.5 \%)$ & 0.58 & .11 & $1.79(0.88-3.63)$ \\
\hline Smoking about a Pack or More Daily in the Past Year ${ }^{b}$ & $62(34.4 \%)$ & $12(26.1 \%)$ & 0.31 & .43 & $1.36(0.63-2.95)$ \\
\hline FTND (moderate-to-high dependence) ${ }^{c}$ & $42(26.4 \%)$ & $8(20.0 \%)$ & 0.40 & .38 & $1.49(0.62-3.59)$ \\
\hline \multirow[t]{2}{*}{$\geq 1$ Quit Attempt ${ }^{\mathrm{d}}$} & $217(75.9 \%)$ & $58(63.7 \%)$ & 0.62 & .019 & $1.87(1.11-3.15)$ \\
\hline & Mean $(S D)$ & Mean $(S D)$ & $F$ & $p$ value & $d$ \\
\hline Time to First Cigarette ${ }^{\mathrm{e}}$ & $2.42(1.06)$ & $2.88(1.02)$ & 4.02 & .046 & .45 \\
\hline \multicolumn{6}{|l|}{ Withdrawal Symptoms ${ }^{f}$} \\
\hline Craving & $2.38(1.18)$ & $2.07(1.12)$ & 5.87 & .016 & .28 \\
\hline Difficulty Concentrating & $1.91(1.12)$ & $1.58(0.96)$ & 8.06 & .005 & .34 \\
\hline Restlessness & $2.03(1.19)$ & $1.54(0.91)$ & 18.10 & $<.001$ & .54 \\
\hline
\end{tabular}

FTND = Fagerström Test for Nicotine Dependence. All analyses excluded 31 LNCG participants diagnosed with ADHD at baseline. Cohen's $d=$ difference between group means divided by LNCG SD.

${ }^{\mathrm{a}} \mathrm{ADHD} n=469$, LNCG $n=240$. There is a difference of $n=1$ between the samples analyzed for this report and in Molina et al..$^{23}$ due to different selection of variables across the analyses.

${ }^{\mathrm{b}} \mathrm{ADHD} n=180$, LNCG $n=46$. These analyses were among to daily smokers in the past year only. See Supplement B for analyses of smoking quantity among the full sample.

"ADHD $n=159$, LNCG $n=40$. Participants were instructed to complete the FTND only if they were "currently a daily smoker."

${ }^{\mathrm{d}} \mathrm{ADHD} n=286$, LNCG $n=91$. These analyses were among those who ever smoked at least weekly.

${ }^{\mathrm{e}} \mathrm{ADHD} n=158$, LNCG $n=40$. A lower score on this FTND item indicates a faster time to first cigarette (ie, $1=$ within first $5 \mathrm{~min}, 2=6-30 \mathrm{~min}, 3=31-60 \mathrm{~min}$, $4=$ after $60 \mathrm{~min})$. The absolute value of the $d$ statistic is reported for this finding.

${ }^{\mathrm{f}} \mathrm{ADHD} n=300$, LNCG $n=108$. The same pattern of results emerged when analyses were repeated among those who ever smoked at least weekly.

smokers did not differ by ADHD/LNCG $(p=.38)$. Mean FTND scores also did not differ (ADHD $M=3.09, S D=2.20 ;$ LNCG $M=2.63, S D=2.34 ; p=.36$ ).

Among participants reporting ever smoking at least once per week, the ADHD group reported a higher proportion of one or more quit attempts than the LNCG $(p=.019)$. Despite this, the ADHD group did not appear to have better smoking cessation rates: there were no group differences in rates of nonsmoking in the past year among those who had ever smoked at least once per week (17\% ADHD, 16\% LNCG, $p=.73$ ). Time to first cigarette of the day was shorter for the ADHD group ( $p=.046$ ). Withdrawal symptoms were higher in the ADHD group than the LNCG for craving, difficulty concentrating, and restlessness ( $p$ 's $\leq .016)$ - the latter remained statistically significant after controlling for normal level of restlessness ( $p=.007)$.

\section{Aim 2: Smoking Initiation, Daily Smoking Onset, and Progression from Initiation to Daily Smoking}

Smoking initiation occurred earlier in the ADHD group than the LNCG across assessments, but this did not reach statistical significance after the inclusion of covariates (Wald $\chi^{2}(1)=0.913, p=.34$, Hazard ratio $=1.09, \mathrm{CI}=0.91-1.31)$. Both median and modal ages for smoking initiation were 15.00 years $(S D=4.90)$ for the ADHD group and 16.00 years $(S D=3.92)$ for the LNCG (see Supplement A).

The ADHD group reported an earlier daily smoking onset than the LNCG across assessments (Wald $\chi^{2}(1)=10.80, p=.001$, Hazard ratio $=1.57, \mathrm{CI}=1.20-2.06$ ) (Figure 1). Median age was the same for both groups, 19.00 years $(\mathrm{SD}=3.64$ and 2.59 for ADHD and LNCG, respectively), but modal age was younger for ADHD (18.00 years) versus the LNCG (19.00 years).

The ADHD group progressed more rapidly from smoking initiation to daily smoking than the LNCG across assessments (Wald $\chi^{2}(1)=6.66, p=.01$, Hazard ratio $\left.=1.43, \mathrm{CI}=1.09-1.88\right)$. Whereas the median latency to daily smoking after initiation was 4.00 years for both the ADHD group $(S D=3.54)$ and the LNCG $(S D=3.79)$, the modal latency was 0.00 (ie, the age of daily smoking onset occurred at the same age as smoking initiation) for the ADHD group and 2.00 years for the LNCG. Initiation age as linear and quadratic terms were also entered in subsequent steps, followed by the initiation age (linear and quadratic) interaction with childhood ADHD status. Statistically significant effects emerged for initiation age as a linear term and as a quadratic term ( $p$ 's $<.001)$, indicating that progression to daily smoking was faster for participants who initiated smoking at an older age and that risk for daily smoking increased as a function of older age. For instance, mean latency was 5.60 years $(S D=3.26)$ for participants who initiated smoking at 17 years old or younger and 1.90 years $(S D=2.08)$ for participants who initiated smoking 18 years old or older. The initiation age (linear) by childhood ADHD status interaction was significant $(p=.025)$, indicating that progression to daily smoking was faster for LNCG participants who initiated smoking at an older age than ADHD participants. For instance, mean latency was 5.61 years $(S D=3.28)$ for ADHD participants and $5.29(S D=3.22)$ for LNCG participants who initiated at 17 year-old or younger, but $2.14(S D=2.26)$ for ADHD participants and $1.20(S D=1.23)$ for LNCG participants who initiated at 18 years-old and older. The other interaction was not statistically significant $(p>.10)$.

\section{Aim 3: Daily Smoking as a function of ADHD Symptom Severity Across Development}

Results of our latent growth model of daily smoking from the 2to 16-year follow-ups in children with ADHD showed good fit to the data $\left(\chi^{2}(133)=168.50, p=.02\right.$; CFI $=.993$; RMSEA $=.021$, $\left.\mathrm{CI}_{95}=.008-.030\right)$. Table 2 shows that daily smoking increased through adolescence $(B=.195, p<.001)$, from an initial probability 


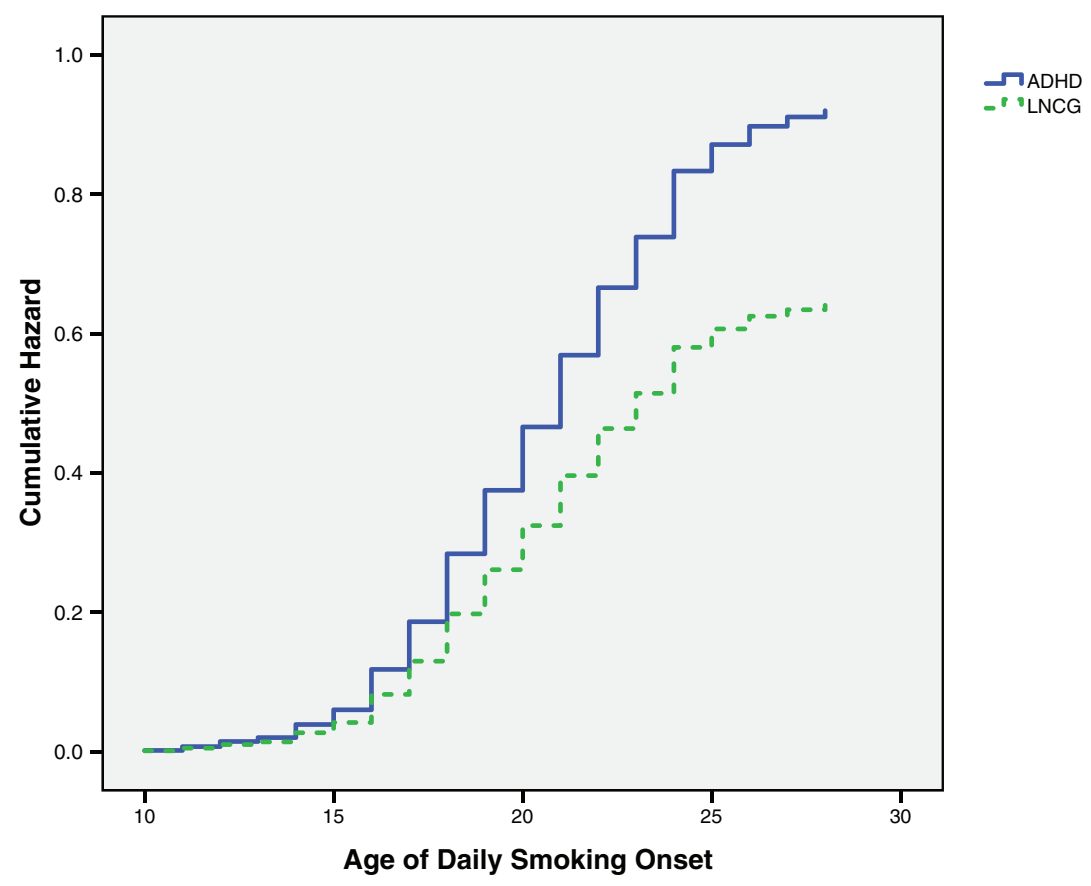

Figure 1. Onset to daily smoking across assessments between groups.

of $0.8 \%$ at the 2 -year follow-up to $33 \%$ by the 12 -year follow-up. The rate of change in daily smoking remained stable through early adulthood $(B=.019, p=.260)$, reaching a predicted base rate of $36 \%$ at the 16 -year follow-up. ADHD symptom severity was a potent correlate of daily smoking at all assessments. Figure 2 shows that at the 16-year follow-up, ADHD symptoms one standard deviation above average predicted a $47 \%$ chance of daily smoking, compared to $25 \%$ for symptoms one standard deviation below average. The main effect of time-varying symptom severity was stable at the adult 12 - to 16 -year follow-ups $(B=.447, p<.001)$, and a symptom $x$ time interaction showed a marginally strengthening association through adolescence $(B=.036, p=.089)$. Figure 2 shows that from the 2- to 12-year follow-ups, ADHD symptoms became more strongly associated with daily smoking every year.

In sensitivity analyses, we explored whether time-varying effects of symptom severity differed for inattention versus hyperactiveimpulsive symptoms. We replaced our composite symptom severity measure with domain-specific symptoms and tested their effects separately. Inattention and hyperactivity-impulsivity were each strongly associated with daily smoking during the adult follow-ups $(B=.390$, $p<.001$ and $B=.366, p<.001$ for inattention and hyperactivityimpulsivity, respectively). Although a symptom $\times$ time interaction involving inattention was statistically significant, the interaction for hyperactivity-impulsivity was not $(B=.038, p=.048$ for inattention; $B=.027, p=.154$ for hyperactivity-impulsivity).

As summarized in Table 2, the probability of daily smoking at the 16-year follow-up was lower for females (24\%), Black ( $8 \%$ ), and other minority participants ( $9 \%$; vs. $36 \%$ for White males). Daily smoking was more likely among young adults coming from disadvantaged households (single-parent family with no college-educated parents, $62 \%$ ) and less likely from advantaged households at baseline (two-parent family with at least one college-educated parent, $17 \%)$. The probability of daily smoking at the 16-year follow-up did not differ between participants with and without comorbid childhood CD/ODD. The rate of daily smoking increased through adolescence, with no differences by sex or household (dis)advantage. The rate of increase among Black participants was $2.0 \%$ faster per year compared to White participants. The rate of increase among children with an initial comorbid diagnosis of CD or ODD was slower compared to children with ADHD alone (3.4\% per year), owing to their higher rates of use at the earliest wave (all five children who reported daily smoking at the 2-year assessment had a comorbid diagnosis of CD/ODD). In early adulthood, women on average became less likely to smoke $(4.0 \%$ faster reduction per year than men in the probability of daily smoking). There were no differences in the rate of change in daily smoking through adulthood across ethnic groups, between participants with and without comorbid childhood CD/ODD, or by household (dis)advantage.

In contrast to the dimensional approach assessing ADHD symptom severity over time, we also considered ADHD persistence categorically using DSM-5 symptom count criteria in adulthood, similar to Hechtman et al. ${ }^{16}$ We found that $43.3 \%$ of the persistent ADHD group reported daily smoking compared to $33.6 \%$ of the desistent ADHD group ( $p=.057$ ) (see Table 1 for LNCG comparison).

\section{Discussion}

This study was a longitudinal analysis of a multisite sample of children comprehensively diagnosed with ADHD and followed over eight assessment points into their mid-20s when smoking behaviors become more firmly established. Our findings indicate that, by adulthood, those with childhood ADHD endorse higher rates of daily cigarette smoking, no differences in smoking cessation rates despite higher rates of quitting at least once, shorter time to first cigarette of the day (an index of greater likelihood of relapse during quit attempts ${ }^{33}$ ), and more severe smoking withdrawal symptoms. Collectively, these findings are novel for a prospectively followed 
Table 2. Latent Growth Model Results Predicting Level and Rates of Change in Daily Smoking Among Participants With ADHD Histories From the 2-Through 16-Year Follow-ups

\begin{tabular}{|c|c|c|}
\hline & $B$ & SE \\
\hline $\begin{array}{l}\text { Time-varying effect of ADHD symptom severity, } 12 \\
\text { to } 16 \text { years }\end{array}$ & $.447^{*}$ & .086 \\
\hline $\begin{array}{l}\text { Rate of change in symptom-smoking relation from } \\
2 \text { to } 12 \text { years }^{\mathrm{a}}\end{array}$ & $.036^{\dagger}$ & .021 \\
\hline \multicolumn{3}{|c|}{$\begin{array}{l}\text { Effects on predicted base rate of daily smoking at 16-year follow-up } \\
(\text { mean age } 25)^{\mathrm{b}}\end{array}$} \\
\hline Female $^{c}$ & $-.350^{*}$ & .150 \\
\hline \multicolumn{3}{|l|}{ Race $^{d}$} \\
\hline Black & $-.1 .021 *$ & .388 \\
\hline Other & $-.981 *$ & .377 \\
\hline $\mathrm{CD} / \mathrm{ODD}$ & .022 & .110 \\
\hline Household advantage & $-.583 *$ & .161 \\
\hline Household disadvantage & $.677 *$ & .204 \\
\hline $\begin{array}{l}\text { Rate of change in daily smoking from Years } 2-12 \\
\text { (Piece } 1 \text { Slope) }\end{array}$ & $.195 *$ & .024 \\
\hline \multicolumn{3}{|l|}{ Race } \\
\hline Black & $.052 *$ & .025 \\
\hline Other & -.040 & .035 \\
\hline $\mathrm{CD} / \mathrm{ODD}$ & $-.085^{*}$ & .014 \\
\hline $\begin{array}{l}\text { Rate of change in daily smoking from Years } 12-16 \\
\text { (Piece } 2 \text { Slope) }\end{array}$ & .019 & .017 \\
\hline Female & $-.095 \%$ & .031 \\
\hline
\end{tabular}

$B=$ Probit regression coefficient (represents unit change in the expected value of a continuous, latent propensity to be a daily smoker on a cumulative standard normal distribution); $S E$ = Standard Error; Analyses were also conducted controlling for Site and Site $\times$ ADHD symptom interactions, which did not produce any effects. ${ }^{*} p<.05 .{ }^{\dagger} p=.089$. ${ }^{a}$ Baseline association (null) between symptom severity and daily smoking at 2 years: $B=.092, S E=.19$. 'Intercept (predicted base rate) of daily smoking at 16-year follow-up: $B=-.367$ $(p<.053), S E=.190$, prob $=.36$. ${ }^{\circ}$ Negative value indicates women are less likely to be daily smokers (Female $=1$, Male $=0)$. ${ }^{\mathrm{d} D u m m y}$ coded: Black $($ Black $=1$, All other $=0)$, Other (Hispanic, other, and mixed ethnicity $=1$, All other $=0$ ). The reference category is White.

CD/ODD (Comorbid CD or ODD diagnosis in childhood=1, No comorbidity $=0$

sample diagnosed with ADHD in childhood. We speculate that greater withdrawal severity in the ADHD group is particularly relevant in the context of the results indicating greater difficulty quitting. Additional findings were that group differences for daily smoking onset and progression to smoking were replicated, which extend past ADHD longitudinal studies in an older cohort. The current study was also the first to demonstrate that ADHD symptom severity was associated with probability of daily smoking across adolescent and adulthood assessments, which suggests that management of ADHD symptoms over development may affect daily smoking rates. We expand on these findings below.

\section{Smoking Outcomes in Young Adulthood}

Young adults with childhood ADHD experienced mixed smoking outcomes. Rates of daily smoking were high (also reported in Molina et $a .^{23}$ ), which extends prior findings to a multisite sample. ${ }^{7,13,42}$ Contrary to cross-sectional findings, ${ }^{43}$ the quantity of cigarettes smoked among daily smokers did not differ between groups. However, this finding does replicate results from a single-site study that included younger participants at follow-up. ${ }^{13}$ One possibility for these null findings is that group differences among daily smokers may emerge as individuals continue to age into their 30s. Another possibility is that cigarette smokers with a history of ADHD may less accurately report on their smoking behavior. In addition to cigarette smokers' inaccuracy recalling specific aspects of smoking behavior, including the number of cigarettes smoked, ${ }^{44}$ individuals with a history of ADHD are often inaccurate reporters of their own behavior. ${ }^{45}$ Future studies of smoking in real time, such as ecological momentary assessments, could address this methodological limitation. These explanations should be examined prior to concluding that smoking quantity is not higher among daily smokers with a history of childhood ADHD.

Nicotine dependence did not differ between the ADHD group and LNCG. These findings are consistent with some, ${ }^{13}$ but not other longitudinal studies of ADHD cohorts that either included nonsmokers ${ }^{46}$ or smaller sample sizes. ${ }^{47}$ Mean scores on the same measure (ie, FTND) were comparable in other ADHD cohorts, ${ }^{13}$ but relatively lower than national trends. ${ }^{48}$ Overall, these findings suggest that children with well-characterized ADHD, and prospectively followed to smoker status in adulthood, have similar levels of nicotine dependence as their peers without a history of ADHD. In contrast, the ADHD group reported more severe withdrawal symptoms (ie, craving, difficulty concentrating, and restlessness). Here again, findings from the MTA converge with Rhodes and colleagues' report that also found greater difficulty concentrating and craving during periods of abstinence. ${ }^{13}$ Together, these parallel findings across two prospectively followed ADHD cohorts may indicate that rates of nicotine dependence are not higher in ADHD, but ADHD is more relevant when withdrawal symptoms emerge following periods of smoking abstinence.

Results pertaining to aspects of smoking cessation were among the most novel findings of adult smoking outcomes that have yet to be identified in prospective studies of childhood diagnosed ADHD. Adult smokers with childhood ADHD more often reported attempting to quit at least once compared to the LNCG. In contrast to previous research, ${ }^{13}$ we may have detected this difference because our participants were older, which extended the timeframe for observation of quit attempts. We also found that despite differences in quit attempts, the ADHD group did not appear to have better smoking cessation rates-this suggests that the ADHD group was more likely to relapse when they attempted to quit. This interpretation is consistent with the finding that the ADHD group had a shorter time to first cigarette, which is a potent predictor of smoking relapse. ${ }^{33}$

More failed quit attempts for adults with ADHD histories may reflect their increased difficulties with withdrawal symptoms, particularly when those symptoms appear to be an unmasking of ADHD symptoms previously "treated" by nicotine. Therefore, our findings of greater withdrawal experience by ADHD could be due to the reemergence of baseline ADHD severity during abstinence. However, laboratory-based protocols where change in baseline ADHD symptoms are examined against smoking withdrawal symptoms over the same period of time during biochemically confirmed smoking abstinence show that withdrawal symptoms are more severe and independent of ADHD symptoms. ${ }^{49}$ The current study was not designed to address this issue, but our findings converge with cross-sectional studies that withdrawal is more severe.

More quit attempts should allow for more opportunities to experience withdrawal symptoms, which are more severe in those with ADHD. This implies a greater number of instances in which cigarette smoking 


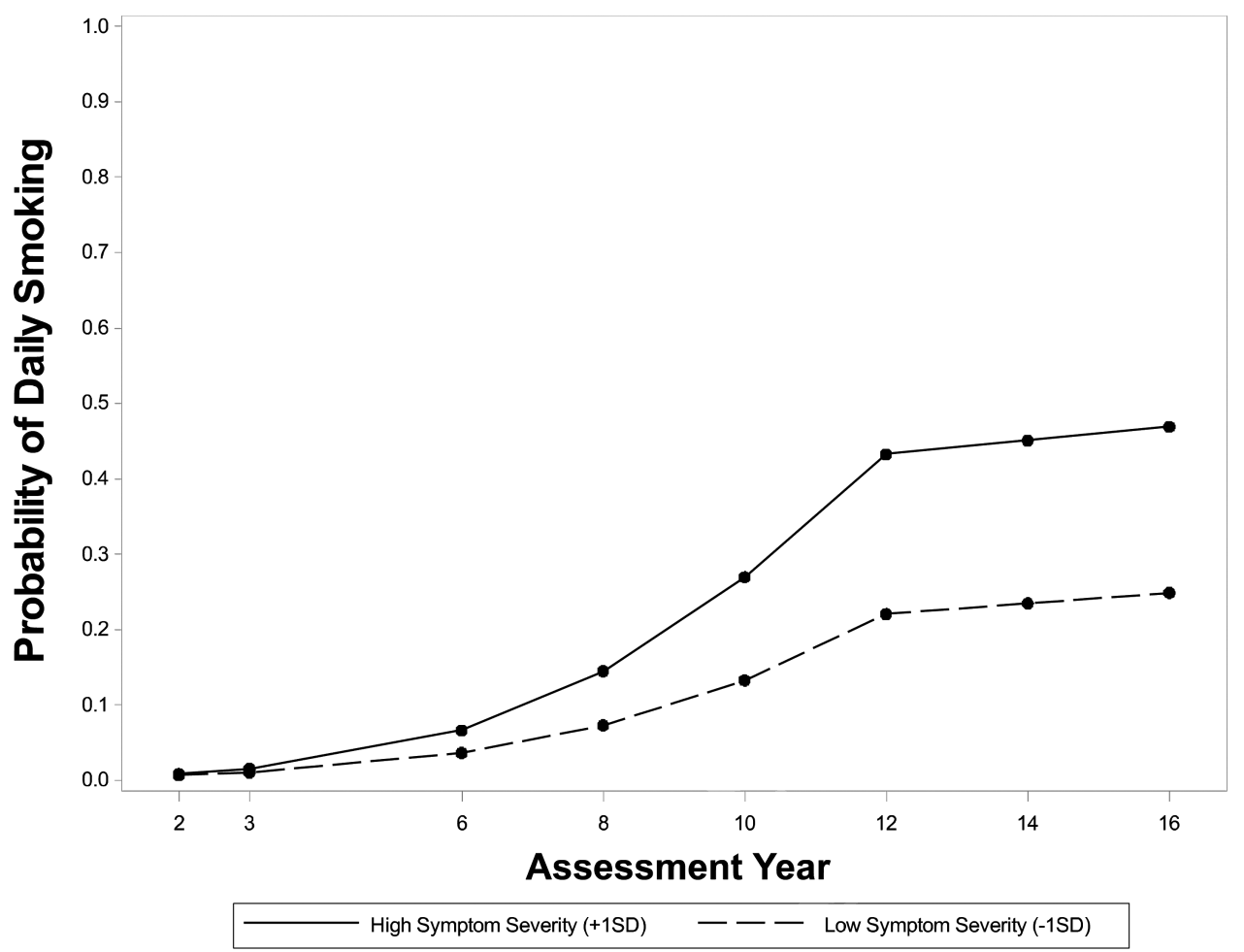

Figure 2. Model-implied probabilities of daily smoking for high and low levels of time-varying ADHD symptom severity, 2- through 16-year assessments, for the childhood ADHD group. $+1 \mathrm{SD}=$ one standard deviation above mean symptom severity, $-1 \mathrm{SD}=$ one standard deviation below mean symptom severity. At any given assessment point, higher-than-usual symptom severity predicts a shift to a trajectory on which daily smoking is more likely.

would be negatively reinforced (ie, smoking relieves withdrawal symptoms) in those with ADHD. This may explain why smoking cessation rates are lower in adult samples with retrospectively diagnosed childhood ADHD. ${ }^{50}$ Related to this latter point, the difficulty quitting that characterized our prospectively followed sample is notable because it overcomes limitations of cross-sectional studies of adult samples that relied on retrospective diagnosis of ADHD in childhood.

Future studies are needed to examine relevant topographical aspects of quitting not measured here, such as whether quit attempts were planned and duration of abstinence. In addition, studies are needed to address how quit attempts are associated with smoking cessation rates in ADHD samples. For example, in addition to negative reinforcement processes, pre-existing motivational deficits and delay aversion $^{51}$ in ADHD may make smoking abstinence disproportionally more difficult. Also, ADHD may lower the ability to tolerate aversive emotional and somatic sensations experienced during smoking abstinence (eg, emotion dysregulation), which is consistent with past studies of ADHD smokers. ${ }^{52}$ Smoking cessation treatments for those with a history of ADHD may be improved by targeting these processes.

\section{Smoking Initiation, Daily Smoking Onset, and Progression from Initiation to Daily Smoking}

We extended prior findings of ADHD cohorts followed into adolescence ${ }^{2-4,13,21,22}$ regarding daily smoking onset and progression to daily smoking by prospectively following a sample into early adulthood. Since most adult smokers begin cigarette use by early adulthood, ${ }^{14}$ we were able to complete the cumulative record of smoking progression using prospective data. These findings converge with other longitudinal studies of ADHD samples followed into adulthood. ${ }^{7,53}$ Whereas the ADHD group was not significantly more likely to try cigarettes earlier than the LNCG across assessments, they did initiate daily smoking earlier and progressed from smoking initiation to daily smoking more quickly. These findings are particularly important in light of this population's difficulty with smoking cessation, both in our sample and elsewhere in cross-sectional adult smoking samples. ${ }^{50}$ If children with ADHD begin smoking daily at young ages and have difficulty quitting, then they are potentially exposed to the harmful effects of smoking over more years of their lives. Although this needs to be tested in samples followed further into adulthood, this pattern would likely place smokers with a history of ADHD at greater risk for negative physical health outcomes of smoking (eg, pulmonary, cardiovascular, and peripheral vascular disease $\left.{ }^{54}\right)$.

\section{Daily Smoking as a Function of ADHD Symptom Severity Across Development}

The association between ADHD symptom severity and daily smoking across assessments is a novel finding that extends previous reports that relied on retrospective self-reported symptoms in a nonclinical sample. ${ }^{55}$ Notable strengths of the current study include a prospectively followed sample and ADHD symptoms assessed by collateral reporters. A novel finding was that ADHD symptoms in late childhood were weakly or unrelated to daily smoking, but by late adolescence, participants exhibiting more severe ADHD symptoms had a higher probability of being daily smokers, an effect that remained strong and stable in adulthood. For example, at the 16-year followup, the chance of being a daily smoker for those with elevated ADHD symptoms was $39.6 \%$ in adulthood, compared to $20.3 \%$ for those with low ADHD symptoms within the ADHD group. Consistent with a broader examination of substance use in the MTA, ${ }^{23}$ patterns 
of use increased in adolescence and became relatively stabilized by young adulthood. These findings implicate the importance of ADHD symptom management into late adolescence and young adulthood on daily smoking. Our sensitivity analyses examining differential effects of ADHD symptoms build on previous studies (see Flory et al., ${ }^{56}$ for a review) and indicate that inattentive symptoms may be particularly important for the development of daily smoking in late adolescence. Although prior studies have demonstrated stronger effects for hyperactive-impulsive symptoms in the early stages of smoking, ${ }^{57}$ inattentive symptoms may play a more prominent role in progression to daily use due to the attention-enhancing effects of nicotine. By adulthood, however, after withdrawal symptoms become more pronounced, all ADHD symptom difficulties may be reflected in the experience of withdrawal (eg, restlessness). Although we caution against over-interpreting the modestly different findings for inattention versus hyperactivity-impulsivity (ie, the symptom domain by time interaction terms yielded beta values that differed by .011 units), the exclusion of children with differential elevations in symptom domains, particularly those with the inattentive subtype of ADHD, should have made this finding harder to detect.

A few effects of demographic covariates emerged. Women were less likely to smoke as adults, and Black adolescents progressed to daily smoking at a faster rate than other adolescents. Those with baseline ODD or CD were slower to progress to daily smoking in adolescence due to higher rates of daily smoking at the earliest assessment. At the 16-year follow-up, those from single-parent families with no college-educated parents were more likely to smoke than those from two-parent families with at least one college-educated parent. These variables need to be considered in greater detail in future studies.

\section{Limitations}

Future studies should also consider limitations not already identified. First, smoking status was not biochemically verified. Second, ADHD combined type was the only subtype allowed at study entry. Those with the inattentive type in childhood may have different smoking profiles. For example, daily smoking onset may be different in a sample with the inattentive type at baseline. Third, future research on reasons for $\mathrm{ADHD}$ vulnerability to daily smoking may benefit from considering the role of parental smoking and in-utero exposure that could affect rates of smoking. Fourth, though we considered baseline ODD and CD, later diagnosis may differentially affect the relationship between childhood ADHD and smoking. Future studies should consider timing of ODD and CD diagnosis and impact on smoking behavior. ${ }^{58}$ Finally, our analyses did not consider stimulant medication effects. Although meta-analytic results do not support that stimulant treatment for ADHD increases the odds of daily smoking in adulthood, ${ }^{59}$ future analyses will consider the impact of MTA treatment group status and naturalistic stimulant medication use effects.

\section{Summary}

This study examined adult outcomes of children with and without ADHD into their mid-20s and is one of the most comprehensive reports to date on a variety of smoking outcomes in a large, prospectively followed sample assessed this far into adulthood. Findings suggest that ADHD in childhood confers a risk for earlier daily smoking onset, faster progression from smoking initiation to daily smoking, more attempts to quit at least once, greater likelihood of relapse during quit attempts, and more severe withdrawal. Further, greater symptom severity into adulthood was associated with greater likelihood of being a daily smoker, underscoring the need for future mechanistic research to examine how ADHD into adolescence and adulthood—not just childhood ADHD—contributes to smoking outcomes. Continued study of these processes is needed to improve efforts to prevent and treat habitual cigarette use among individuals with ADHD.

\section{Funding}

This work was supported by cooperative agreement grants and contracts from National Institute of Mental Health (NIMH) and the National Institute on Drug Abuse (NIDA) at the National Institutes of Health (NIH) to the following: University of California-Berkeley: U01 MH50461, N01MH12009, HHSN271200800005-C, and DA-8-5550; Duke University: U01 MH50477, N01MH12012, HHSN271200800009-C, and DA-8-5554; University of California- Irvine: U01MH50440,N01MH1201, HHSN271200800006-C, and DA-8-5551; Research Foundation for Mental Hygiene (New York State Psychiatric Institute/Columbia University): U01 MH50467, N01 MH12007, HHSN271200800007-C, and DA-8-5552; Long Island-Jewish Medical Center U01 MH50453; New York University: N01MH 12004, HHSN271200800004-C, and DA-8-5549; University of Pittsburgh: U01 MH50467, N01 MH 12010, DA039881, HHSN271200800008-C, and DA8-5553; and McGill University N01MH12008, HHSN271200800003-C, and DA-8-5548. Additional funding support provided by NIDA (K23DA032577 to J.T.M., K24DA023464 to S.H.K., R01DA039881 to B.S.G.M.).

\section{Declaration of Interests}

In the past 2 years: Dr. Mitchell has received royalties from New Harbinger Press; Dr. Belendiuk has stocklequity in Shire and Roche; Dr. Hechtman has received research funding, served on the advisory boards and has been speaker for Ely Lilly, GlaxoSmithKline, Ortho Janssen, Purdue, and Shire; Dr. Arnold has received research funding from Curemark, Forest, Lilly, Neuropharm, Novartis, Noven, Shire, Supernus, and YoungLiving (as well as NIH and Autism Speaks) and has consulted with or been on advisory boards for Arbor, Gowlings, Neuropharm, Novartis, Noven, Organon, Otsuka, Pfizer, Roche, Seaside Therapeutics, Sigma Tau, Shire, Tris Pharma, and Waypoint; Dr. Kollins has received research support and/or consulting fees from the following: Akili Interactive, Alcobra, Arbor, Atentiv, CogCubed, Kempharm, Intelligent Automation, Ironshore, Neos, NIH, Purdue Canada, Rhodes, Shire, Sunovion, Tris, and SK Life Sciences. Dr. Vitiello has served as consultant to the law firm Gowling WLG, Ottawa, Canada. None of the other authors have any additional declarations.

\section{Acknowledgments}

The Multimodal Treatment Study of Children with ADHD (MTA) was a National Institute of Mental Health (NIMH) cooperative agreement randomized clinical trial, continued under an NIMH contract as a follow-up study and finally under a National Institute on Drug Abuse (NIDA) contract. Collaborators from NIMH: Benedetto Vitiello (Child \& Adolescent Treatment and Preventive Interventions Research Branch), Joanne B. Severe (Clinical Trials Operations and Biostatistics Unit, Division of Services and Intervention Research), Peter S. Jensen (currently at REACH Institute and Mayo Clinic), L. Eugene Arnold (currently at Ohio State University), Kimberly Hoagwood (currently at Columbia); previous contributors from NIMH to the early phases: John Richters (currently at National Institute of Nursing Research); Donald Vereen (currently at NIDA). Principal investigators and co-investigators from the sites are: University of California, Berkeley/San Francisco: Stephen P. Hinshaw (Berkeley), Glen R. Elliott (San Francisco); Duke University: Karen C. Wells, Jeffery N. Epstein (currently at Cincinnati Children's Hospital Medical Center), Desiree W. Murray; previous Duke contributors to early phases: C. Keith Conners (former PI); John March; University of California, Irvine: James Swanson, Timothy Wigal; previous contributor from UCLA to the early phases: Dennis P. Cantwell (deceased); New York University School of Medicine: Howard B. Abikoff; Montreal Children's Hospital/ McGill University: Lily Hechtman; New York State Psychiatric Institute/Columbia 
University/Mount Sinai Medical Center: Laurence L. Greenhill (Columbia), Jeffrey H. Newcorn (Mount Sinai School of Medicine). University of Pittsburgh: Brooke Molina, Betsy Hoza (currently at University of Vermont), William E. Pelham (PI for early phases, currently at Florida International University). Follow-up phase statistical collaborators: Robert D. Gibbons (University of Illinois, Chicago); Sue Marcus (Mt. Sinai College of Medicine); Kwan Hur (University of Illinois, Chicago). Original study statistical and design consultant: Helena C. Kraemer (Stanford University). Collaborator from the Office of Special Education Programs/US Department of Education: Thomas Hanley. Collaborator from the Office of Juvenile Justice and Delinquency Prevention/ Department of Justice: Karen Stern. Appreciation is extended to Saul Shiffman, for his guidance regarding the development of the cigarette smoking section of the Substance Use Questionnaire.

\section{References}

1. American Psychiatric Association. Diagnostic and Statistical Manual of Mental Disorders, 5th edition (DSM-5). Washington, D.C.: American Psychiatric Association; 2013.

2. Molina BS, Pelham WE Jr. Childhood predictors of adolescent substance use in a longitudinal study of children with ADHD. J Abnorm Psychol. 2003;112(3):497-507.

3. Milberger S, Biederman J, Faraone SV, Chen L, Jones J. ADHD is associated with early initiation of cigarette smoking in children and adolescents. J Am Acad Child Adolesc Psychiatry. 1997;36(1):37-44.

4. Sibley MH, Pelham WE, Molina BSG, et al. The role of early childhood ADHD and subsequent $\mathrm{CD}$ in the initiation and escalation of adolescent cigarette, alcohol, and marijuana use. J Abnorm Psychol. 2014;123(2):362-374.

5. Biederman J, Monuteaux MC, Mick E, et al. Young adult outcome of attention deficit hyperactivity disorder: A controlled 10-year follow-up study. Psychol Med. 2006;36(2):167-179.

6. Biederman J, Petty CR, Woodworth KY, Lomedico A, Hyder LL, Faraone SV. Adult outcome of attention-deficit/hyperactivity disorder: A controlled 16-year follow-up study. J Clin Psychiatry. 2012;73(7):941-950.

7. Lambert NM, Hartsough CS. Prospective study of tobacco smoking and substance dependencies among samples of ADHD and non-ADHD participants. J Learn Disabil. 1998;31(6):533-544.

8. Lambert N. The contribution of childhood ADHD, conduct problems, and stimulant treatment to adolescent and adult tobacco and psychoactive substance abuse. Ethical Hum Psychol Psychiatry. 2005;7(3):197-221.

9. Klein RG, Mannuzza S, Olazagasti MA, et al. Clinical and functional outcome of childhood attention-deficit/hyperactivity disorder 33 years later. Arch Gen Psychiatry. 2012;69(12):1295-1303.

10. Wilens TE, Vitulano M, Upadhyaya H, et al. Concordance between cigarette smoking and the modified Fagerström Tolerance Questionnaire in controlled studies of ADHD. Am J Addict. 2008;17(6):491-496.

11. Brook JS, Duan T, Zhang C, Cohen PR, Brook DW. The association between attention deficit hyperactivity disorder in adolescence and smoking in adulthood. Am J Addict. 2008;17(1):54-59.

12. Orlando M, Tucker JS, Ellickson PL, Klein DJ. Developmental trajectories of cigarette smoking and their correlates from early adolescence to young adulthood. J Consult Clin Psychol. 2004;72(3):400-410.

13. Rhodes JD, Pelham WE, Gnagy EM, Shiffman S, Derefinko KJ, Molina BS. Cigarette smoking and ADHD: An examination of prognostically relevant smoking behaviors among adolescents and young adults. Psychol Addict Behav. 2016;30(5):588-600.

14. The Health Consequences of Smoking-50 Years of Progress: A Report of the Surgeon General. Atlanta, GA: US Department of Health and Human Services, Centers for Disease Control and Prevention, National Center for Chronic Disease Prevention and Health Promotion, Office of Smoking and Health; 2014.

15. Sibley MH, Swanson JM, Arnold LE, et al.; MTA Cooperative Group. Defining ADHD symptom persistence in adulthood: Optimizing sensitivity and specificity. J Child Psychol Psychiatry. 2017;58(6):655-662.

16. Hechtman L, Swanson JM, Sibley MH, et al.; MTA Cooperative Group. Functional adult outcomes 16 years after childhood diagnosis of attention-deficit/hyperactivity disorder: MTA results. J Am Acad Child Adolesc Psychiatry. 2016;55(11):945-952.e2.

17. Sibley MH, Pelham WE, Molina BSG, et al. When diagnosing ADHD in young adults emphasize informant reports, DSM items, and impairment. $J$ Consult Clin Psychol. 2012;80(6):1052-1061.

18. Faraone SV, Kunwar A, Adamson J, Biederman J. Personality traits among ADHD adults: Implications of late-onset and subthreshold diagnoses. Psychol Med. 2009;39(4):685-693.

19. Bjartveit K, Tverdal A. Health consequences of smoking 1-4 cigarettes per day. Tob Control. 2005;14(5):315-320.

20. MTA Cooperative Group. A 14-month randomized clinical trial of treatment strategies for attention-deficit/hyperactivity disorder. The MTA cooperative group. Multimodal Treatment Study of Children with ADHD. Arch Gen Psychiatry. 1999;56(12):1073-1086.

21. Molina BS, Flory K, Hinshaw SP, et al. Delinquent behavior and emerging substance use in the MTA at 36 months: Prevalence, course, and treatment effects. J Am Acad Child Adolesc Psychiatry. 2007;46(8):1028-1040.

22. Molina BS, Hinshaw SP, Eugene Arnold L, et al.; MTA Cooperative Group. Adolescent substance use in the multimodal treatment study of attention-deficit/hyperactivity disorder (ADHD) (MTA) as a function of childhood ADHD, random assignment to childhood treatments, and subsequent medication. J Am Acad Child Adolesc Psychiatry. 2013;52(3):250-263.

23. Molina BSG, Howard AL, Swanson JM, et al. Substance use through adolescence into early adulthood after childhood-diagnosed ADHD: Findings from the MTA longitudinal study. J Child Psychol Psychiatry. 2018 (In this issue).

24. MTA Cooperative Group. Moderators and mediators of treatment response for children with attention-deficit/hyperactivity disorder: The Multimodal Treatment Study of children with Attention-deficit/hyperactivity disorder. Arch Gen Psychiatry. 1999;56(12):1088.

25. Molina BS, Hinshaw SP, Swanson JM, et al.; MTA Cooperative Group. The MTA at 8 years: Prospective follow-up of children treated for combinedtype ADHD in a multisite study. J Am Acad Child Adolesc Psychiatry. 2009;48(5):484-500.

26. Howard AL, Strickland NJ, Murray DW, et al. Progression of impairment in adolescents with attention-deficit/hyperactivity disorder through the transition out of high school: Contributions of parent involvement and college attendance. J Abnorm Psychol. 2016;125(2):233-247.

27. Swanson JM, Arnold LE, Molina BSG, et al.; MTA Cooperative Group. Young adult outcomes in the follow-up of the multimodal treatment study of attention-deficit/hyperactivity disorder: Symptom persistence, source discrepancy, and height suppression. J Child Psychol Psychiatry. 2017;58(6): 663-678.

28. Molina BS, Pelham WE Jr. Childhood predictors of adolescent substance use in a longitudinal study of children with ADHD. J Abnorm Psychol. 2003;112(3):497-507.

29. Schane RE, Ling PM, Glantz SA. Health effects of light and intermittent smoking: A review. Circulation. 2010;121(13):1518-1522.

30. Heatherton TF, Kozlowski LT, Frecker RC, Fagerström KO. The fagerström test for nicotine dependence: A revision of the Fagerström tolerance questionnaire. Br J Addict. 1991;86(9):1119-1127.

31. Japuntich SJ, Piper ME, Schlam TR, Bolt DM, Baker TB. Do smokers know what we're talking about? The construct validity of nicotine dependence questionnaire measures. Psychol Assess. 2009;21(4):595-607.

32. Weinberger AH, Reutenauer EL, Allen TM, et al. Reliability of the Fagerström test for nicotine dependence, Minnesota nicotine withdrawal scale, and tiffany questionnaire for smoking urges in smokers with and without schizophrenia. Drug Alcohol Depend. 2007;86(2-3):278-282.

33. Baker TB, Piper ME, McCarthy DE, et al. Time to first cigarette in the morning as an index of ability to quit smoking: Implications for nicotine dependence. Nicotine Tob Res. 2007;9 Suppl 4:S555-570.

34. Swanson J. SNAP-IV Scale. Irvine, CA: University of California Child Development Center; 1995.

35. Conners CK, Erhardt D, Sparrow E. Conners' Adult ADHD Rating Scales (CAARS) technical manual. North Tonawanda, NY: Multi-Health Systems, Inc; 1999. 
36. Solanto MV, Alvir J. Reliability of DSM-IV symptom ratings of ADHD: Implications for DSM-V. J Atten Disord. 2009;13(2):107-116.

37. Nichols JQ, Shoulberg EK, Garner AA, et al. Exploration of the factor structure of ADHD in adolescence through self, parent, and teacher reports of symptomatology. J Abnorm Child Psychol. 2017;45(3):625-641.

38. Molina BSG, Pelham WE, Cheong J, Marshal MP, Gnagy EM, Curran PJ. Childhood attention-deficit/hyperactivity disorder (ADHD) and growth in adolescent alcohol use: The roles of functional impairments, ADHD symptom persistence, and parental knowledge. J Abnorm Psychol. 2012;121(4):922-935.

39. Muthén LK, Muthén BO. Mplus User's Guide. $6^{\text {th }}$ ed. Los Angeles, CA: Muthén \& Muthén; 2010.

40. Flora DB. Specifying piecewise latent trajectory models for longitudinal data. Struct Equ Modeling. 2008;15(3):513-533.

41. Asparouhov T, Muthen B. Weighted Least Squares Estimation with Missing Data. 2010; http://www.statmodel.com/download/ GstrucMissingRevision.pdf.

42. Wilens TE, Martelon M, Joshi G, et al. Does ADHD predict substance-use disorders? A 10-year follow-up study of young adults with ADHD. J Am Acad Child Adolesc Psychiatry. 2011;50(6):543-553.

43. Kollins SH, McClernon FJ, Fuemmeler BF. Association between smoking and attention-deficit/hyperactivity disorder symptoms in a population-based sample of young adults. Arch Gen Psychiatry. 2005;62(10):1142-1147.

44. Shiffman S, Hufford M, Hickcox M, Paty JA, Gnys M, Kassel JD. Remember that? A comparison of real-time versus retrospective recall of smoking lapses. J Consult Clin Psychol. 1997;65(2):292-300.

45. Sibley MH, Pelham WE, Molina BSG, et al. Diagnosing ADHD in adolescence. J Consult Clin Psychol. 2012;80(1):139-150.

46. Groenman AP, Oosterlaan J, Rommelse N, et al. Substance use disorders in adolescents with attention deficit hyperactivity disorder: A 4-year follow-up study. Addiction. 2013;108(8):1503-1511.

47. Wilens TE, Vitulano M, Upadhyaya $\mathrm{H}$, et al. Cigarette smoking associated with attention deficit hyperactivity disorder. $J$ Pediatr. 2008;153(3):414-419.

48. Fagerström K, Furberg H. A comparison of the Fagerström test for nicotine dependence and smoking prevalence across countries. Addiction. 2008;103(5):841-845.
49. McClernon FJ, Van Voorhees EE, English J, Hallyburton M, Holdaway A, Kollins SH. Smoking withdrawal symptoms are more severe among smokers with ADHD and independent of ADHD symptom change: Results from a 12-day contingency-managed abstinence trial. Nicotine Tob Res. 2011;13(9):784-792.

50. Humfleet GL, Prochaska JJ, Mengis M, et al. Preliminary evidence of the association between the history of childhood attention-deficit/hyperactivity disorder and smoking treatment failure. Nicotine Tob Res. 2005;7(3):453-460.

51. Sonuga-Barke EJ. Causal models of attention-deficit/hyperactivity disorder: From common simple deficits to multiple developmental pathways. Biol Psychiatry. 2005;57(11):1231-1238.

52. Mitchell JT, Dennis MF, English JS, et al. Ecological momentary assessment of antecedents and consequences of smoking in adults with attention-deficit/hyperactivity disorder. Subst Use Misuse. 2014;49(11):1446-1456.

53. Rohde P, Kahler CW, Lewinsohn PM, Brown RA. Psychiatric disorders, familial factors, and cigarette smoking: II. Associations with progression to daily smoking. Nicotine Tob Res. 2004;6(1):119-132.

54. Centers for Disease Control and Prevention. The Health Consequences of Smoking: A Report of the Surgeon General. Washington, DC: Department of Health and Human Services, Centers for Disease Control and Prevention, National Center for Chronic Disease Prevention and Health Promotion, Office of Smoking and Health: 2004.

55. Fuemmeler BF, Kollins SH, McClernon FJ. Attention deficit hyperactivity disorder symptoms predict nicotine dependence and progression to regular smoking from adolescence to young adulthood. J Pediatr Psychol. 2007;32(10):1203-1213.

56. Glass K, Flory K. Why does ADHD confer risk for cigarette smoking? A review of psychosocial mechanisms. Clin Child Fam Psychol Rev. 2010;13(3):291-313.

57. Chang Z, Lichtenstein P, Larsson H. The effects of childhood ADHD symptoms on early-onset substance use: A Swedish twin study. J Abnorm Child Psychol. 2012;40(3):425-435.

58. Groenman AP, Janssen TWP, Oosterlaan J. Childhood psychiatric disorders as risk factor for subsequent substance abuse: A meta-analysis. J Am Acad Child Adolesc Psychiatry. 2017;56(7):556-569.

59. Schoenfelder EN, Faraone SV, Kollins SH. Stimulant treatment of ADHD and cigarette smoking: A meta-analysis. Pediatrics. 2014;133(6):1070-1080. 